\title{
An artificial Kepler dichotomy? Implications for the coplanarity of planetary systems
}

\author{
Timothy Bovaird ${ }^{1}$ and Charles H. Lineweaver ${ }^{1,2}$ \\ ${ }^{1}$ Research School of Astronomy and Astrophysics, Australian National University, Canberra, \\ ACT 2611, Australia \\ ${ }^{2}$ Research School of Earth Sciences, Australian National University
}

\begin{abstract}
We challenge the assumptions present in previous efforts to model the ensemble of detected Kepler systems, which require a dichotomous stellar population of 'fertile' and 'sterile' planet producing stars. We remove the assumption of Rayleigh distributed mutual inclinations between planets and show that the need for two distinct stellar populations disappears when the inner part of planetary disks are assumed to be flat, rather than flared.
\end{abstract}

Keywords. Kepler, multiple-planet systems, coplanarity, protoplanetary disks

\section{The Kepler Dichotomy}

The Kepler dichotomy is an inference drawn from modeling the number of Kepler detected planetary systems. While the simulated populations are well matched by the detected sample, the number of systems with a single transiting planet is underestimated by a factor of $\sim 3$ (Lissauer et al. (2011), Johansen et al. (2012), and Ballard \& Johnson (2014)). These studies propose that the Kepler stellar sample is dichotomous in its planet producing capability, separated into stars which produce many planets, and stars which largely produce a single planet.

\section{Model Dependence}

An assumption in the above studies is that the mutual inclinations between the orbital plane's of planets are Rayleigh distributed. This is equivalent to a flared disk, where a planet's vertical distance from the invariable plane is proportional to its semi-major axis. In our Solar System, this is only seen exterior to the orbit of Jupiter, and the vertical height above the invariable plane is approximately constant for the inner planets. We propose using a new disk model for our simulated Kepler systems, a 'flat' disk model where a planet's height above the invariable plane is independent of semi-major axis.

Another assumption in the simulated systems is that the number of planets in a given system can be drawn from a Poisson distribution. This implies that planet formation within a given system in an independent process. For our simulated Kepler systems, we trial a planet formation process where planets in the inner disk are formation sequentially Chatterjee \& Tan (2014). This results in an exponential multiplicity distribution.

Under these new assumptions, our simulations show that the Kepler stellar sample can be represented by a single stellar population with respect to planet formation. The requirement of a dichotomous stellar population with distinct fertile and sterile planetary formation properties is either significantly reduced, or removed entirely.

\section{References}

Lissauer, J. J. et al. 2011, ApJS, 197, 8

Johansen, A., Davies, M. B., Church, R. P., \& Holmelin, V. 2012, ApJ, 758, 39

Ballard, S. \& Johnson, J. A. 2014, ApJ, submitted

Chatterjee, S., \& Tan, J. C., 2014, ApJ 780, 53 